\title{
Title: Evaluation of correlation between left atrial pressure and left ventricular end diastolic pressure in patients with mitral regurgiation
}

Authors: Jafar Golshahi, MD, internist \& cardiologist

Amir Raufi MD, resident of cardiology

Affiliation: Department of Internal Medicine, Isfahan Medical School

\section{Introduction:}

Left Ventricular End Diastolic Pressure (LVEDP) is of the important hemodynamic variables that is important in determination of cardiac function so that it increases both in systolic and diastolic left ventricular dysfunction(1). Measurement of LVEDP can provide the diagnosis of heart failure(2) and the efficacy of drug and non-drug therapy on it(3) and also the effect of other drugs on left ventricular function and the prognosis of cardiac patients(4). The goal of this study is to assess the accuracy of Left Atrial Pressure (LAP) measured by Doppler echocardiography in LVEDP estimation.

\section{Methods:}

In this study, 25 candidates of left ventricular catheterization with any indication who had mitral valve regurgitation $\left(1^{+}\right.$or more) confirmed with ventriculography in catheterization were selected. A written consent was obtained from every individual. LVEDP was directly measured with a pigtail catheter at left ventriculography and systolic blood pressure with a standard mercury manometer. Then LAP was measured by calculation of MR jet peak velocity assessed in echocardiography and SBP using Bernoulli equation and later with Pearson correlation test, the correlation coefficient between LAP and LVEDP was calculated.

\section{Results:}

Correlation test, with a correlation coefficient of $\mathrm{r}=0.76$ with $\mathrm{P}<0.001$, showed a direct correlation between LAP measured by echocardiography and LVEDP measured by catheterization. This study also showed that on the basis of regression line equation ( $y$ on the basis of $\mathrm{x}$ ), LVEDP on the basis of LAP can be estimated and therefore predictable so that $\mathrm{LVEDP}=0.183+0.851 \mathrm{LAP}$.

\section{Discussion:}

Doppler echocardiography is an acceptable procedure in estimation of LVEDP in patients with MR in comparison to the gold standard procedure of catheterization(1). Therefore, since Doppler echocardiography is available, non-invasive, repeatable and possible at bedside with low complications; it is introduced as an accurate alternative procedure in LVEDP measurement as an easily accessible variable at bedside for evaluation, diagnosis and treatment of patients with heart failure and MR.

\section{References:}

1. Braunwald E, Zipea DP, Libby P, Heart Dis. $6^{\text {th }}$ edition, Philadelphia: W.B. Saunders Company, 2001; 519,481-482, 486, 372.

2. Sharma VRK, et al. evaluation of a noninvasive system for determining left ventricular filling pressure, Arsh Intern Med, 2002; 162:2090-2096. 